\title{
Study on Application of Image Processing For Fertilized Eggs Monitoring
}

\author{
Sheng-Yu Tsai ${ }^{1}$, Ching-Wei Cheng ${ }^{2}$ \\ ${ }^{1}$ Department of Bio-industrial Mechatronics Engineering, National Chung Hsing University \\ 145 Xingda Rd., South Dist., Taichung City 402, Taiwan (R.O.C.) \\ ya.yu1107@gmail.com; cwcheng@nutc.edu.tw \\ ${ }^{2}$ College of Intelligence, National Taichung University of Science and Technology \\ No.129, Sec.3, Sanmin Rd, North Dist., Taichung City 404, Taiwan (R.O.C.)
}

\section{Extended Abstract}

Most influenza vaccines are egg-based vaccines made from eggs. Each fertilized egg is a good independent breeding room after disinfection. That is currently recognized as the safest and most efficient production method. However, three fertilized eggs can only make into a $0.5 \mathrm{ml}$ vaccine. Vaccine manufacturers usually inject seed viruses on the 9th to 12th day of egg hatching. Therefore, the hatchery will detect good fertilized eggs before the 10th day of hatching. At present, most of the methods of detecting fertilized eggs in the hatchery are candling eggs, which uses a bright light source behind the egg to show details through the shell. Recently, numerous non-destructive detection-based studies have been done on monitoring the hatching process of eggs, including machine vision, percussion vibration methods [1], optical detection method, and dielectric characteristic measurement method. In 2016, Hashemzadeh et al. used machine vision to identify whether an egg was fertilized. In their study, an LED lamp was used to penetrate the eggshell at a close range, with a CCD camera used to film the egg's embryo growth [2]. The optical detection method has a wide range of applications in the detection of agricultural products, including the detection of the sugar content, $\mathrm{pH}$ value, and water content [3-5] of agricultural products; of egg products, while whether an egg is fertilized [6,7] is determined by the light absorption ratios of different egg compositions, such as eggshell, egg white, blood vessels, and erythrocytes developed during the embryo growth inside the egg, which exhibit different absorption spectral bands. The purpose of this study is to use image processing instead of manual observation. Not only can effectively reduce human pollution, but also avoid occupational injuries caused by bright light. The research method uses self-made equipment to obtain sample data onto 150 white eggs from the 1st to the 19th. It uses image processing methods and Receiver Operating Characteristic curve statistics to find the key parameters for classifying fertilized eggs, unfertilized eggs, and dead-in-shell embryos. Finally, verify the classification results from 50 eggs. According to the experimental results, using 50 eggs to verify the results, the unfertilized eggs have a detection accuracy of $94 \%$ starting on the 6th day, and the threshold value is 207 . At the same time, the dead-in-shell embryos can reach $96 \%$ on the 9 th day, and the threshold value is 75 . The results show that the best cutoff value established by the average gray value of the red layer of 150 eggs can screen out unfertilized eggs and dead-inshell embryos before the ninth day. Therefore, the method provided in this study can effectively and quickly detect eggs that are not suitable for vaccine production.

\section{References}

[1] Coucke, P. M., Room, G. M., Decuypere, E. M., \& de Baerdemaeker, J. G. 1997. Monitoring embryo development in chicken eggs using acoustic resonance analysis. Biotechnology Progress, 13(4), 474-478.

[2] Hashemzadeh, M., \& Farajzadeh, N. 2016. A machine vision system for detecting fertile eggs in the incubation industry. International Journal of Computational Intelligence Systems, 9(5), 850-862.

[3] Guan, X., Liu, J., Huang, K., Kuang, J. Y., \& Liu, D. D. 2019. Evaluation of moisture content in processed apple chips using NIRS and wavelength selection techniques. Infrared Physics \& Technology, 98, 305-310.

[4] Li, J. B., Huang, W. Q., Zhao, C. J., \& Zhang, B. H. 2013. A comparative study for the quantitative determination of soluble solids content, $\mathrm{pH}$ and firmness of pears by Vis/NIR spectroscopy. Journal of Food Engineering, 116(2), 324332. 
[5] Ying, Y. B., \& Liu, Y. D. 2008. Nondestructive measurement of internal quality in pear using genetic algorithms and FT-NIR spectroscopy. Journal of Food Engineering, 84(2), 206-213.

[6] Bamelis, F. R., Tona, K., de Baerdemaeker, J. G., \& Decuypere, E. M. 2002. Detection of early embryonic development in chicken eggs using visible light transmission. British Poultry Science, 43(2), 204-212.

[7] Dong, J., Dong, X. G., Li, Y. L., Peng, Y. K., Chao, K. L., Gao, C. Y., \& Tang, X. Y. 2019. Identification of unfertilized duck eggs before hatching using visible/near infrared transmittance spectroscopy. Computers and Electronics in Agriculture, 157, 471-478. 